\title{
Factores críticos de éxito turístico para comunidades asentadas en zonas de litoral. Caso de estudio: Comunidad de Salango, provincia de Manabí, Ecuador.
}

\section{Factors for Success community tourism in coastal areas. Study case of Community of Salango, Manabi province, Ecuador.}

Gustavo Xavier Álvaro Silva. ${ }^{1}$, Pablo Aníbal Torres Matovelle. ${ }^{2}$ \& Ramón Martín Fernández. ${ }^{3}$

\section{Resumen.}

El manejo del turismo comunitario es una alternativa auténtica para la mejora de las condiciones de vida de quienes se dedican a esta actividad. Los pueblos asentados en zonas del litoral poseen un gran potencial cultural y natural para el aprovechamiento turístico, pero se requiere emplear variables cuyo propósito sea lograr el éxito a largo plazo de cada emprendimiento comunitario. Ante lo expuesto y con la premisa de que la participación activa de la comunidad en la toma de decisiones es determinante, utilizando la técnica de taller, se puso a consideración de un grupo de representantes del Centro de Turismo Comunitario Salango ubicado en la provincia de Manabí, Ecuador, dieciocho factores claves de éxito que permiten identificar actividades en las que deben prioritariamente centrar su atención.

Palabras claves: factores críticos, éxito turístico, comunidad, Salango.

\section{Abstract.}

The management of community tourism is an authentic alternative for the improvement of the living conditions of those who dedicate themselves to this activity. The people

\footnotetext{
${ }^{1}$ Universidad Laica Eloy Alfaro de Manabí - Manta - Ecuador. gustavo.alvaro@uleam.edu.ec

${ }^{2}$ Universidad de La Habana - Cuba. pablotorresmatovelle@ gmail.com

${ }^{3}$ Universidad de La Habana - Cuba.ramon_martin@ftur.uh.cu
} 
living in coastal areas have great cultural and natural potential for tourism, but it is necessary to use variables which purpose is to achieve the long-term success of each community enterprise. Given the above mentioned and with the premise that the active participation of the community in decision making is determinant, using the workshop technique, was put to consideration of a group of representatives of the Community Tourism Center Salango located in the Manabí province, Ecuador, eighteen key success factors that allow identifying activities in which they must focus their attention first.

Keywords: critical factors, success tourism, community, Salango.

\section{Introducción.}

Las localidades turísticas, sea cual fuere su ubicación geográfica, a fin de obtener el máximo beneficio de sus ventajas comparativas, deben adoptar medidas que día a día posibiliten su empoderamiento exitoso en el concierto turístico global.

Siendo un constructo latente, como lo refiere Torres (2018), el éxito turístico de un destino puede definirse a partir de un conjunto de dimensiones, sobre las cuales, por cierto, no existe consenso académico [1]. En ese sentido el éxito puede tener diferentes significados dependiendo del tipo de destino y los intereses de sus involucrados. Esto determina la necesidad de analizar lo que comprende el éxito para quienes son responsables y beneficiarios del desarrollo turístico.

Entre los instrumentos metodológicos que han sido recurrentemente utilizados para el estudio del éxito cuentan los factores críticos. Inicialmente planteados por Daniel (1961), estos factores han sido ampliamente trabajados en el marco de la gestión estratégica, sirviendo de base incluso para el desarrollo de sistemas como el Balanced Score Card (Kaplan y Norton, 1982) [2]. También han sido utilizados en el plano turístico, especialmente en trabajos relacionados con la competitividad. Ferreras (2009) [3].

Un factor crítico o clave se define como aquel que siendo importante, resulta de extraordinaria relevancia para alcanzar los objetivos planteados. Esto quiere decir que entre un conjunto de elementos que son pertinentes y necesarios para lograr el éxito en un contexto de gestión dado, se diferencian aquellos elementos que son de estricto cumplimiento o dicho de otra manera, aquellos que ostentan una mayor capacidad de contribuir al éxito perseguido.

El enfoque en factores clave de éxito favorece considerablemente al éxito de la posición competitiva de una organización. De Freitas, V; Yaber, G. (2015) [4]. A nivel turístico, el enfoque es especialmente útil en la medida que permite discriminar las acciones de mayor pertinencia de entre un extenso abanico de iniciativas.

Se pretende, con los resultados obtenidos en la presente investigación, contribuir a la mejora de las condiciones de la prestación de los servicios que brindan los gestores de emprendimientos de turismo rural comunitario, sea cual fuere su ubicación geográfica.

\section{Turismo comunitario.}

En Ecuador se entiende por comunidad los lugares donde se materializa la vida de todos los ciudadanos como seres humanos que en ella habitan. Es el resultado de la sumatoria 
de dinámicas y cosmovisiones de ser y pensar el mundo, diversas pero confluyentes que implican pensar un Estado acorde a ellas, para generar espacios de intercambio y encuentro común. Plan Nacional del Buen Vivir (2017) [5].

Partiendo se esta premisa, la comunidad organizada dentro del país está incursionando en el turismo comunitario, que por su propia naturaleza se desarrolla en zonas rurales, cuya evolución ha sido favorable, si se parte del enfoque de la Federación Ecuatoriana Plurinacional de Turismo Comunitario - FEPTCE que expresa que éste se basa en una forma de vida que corresponde a ciertas dinámicas de organización social, en donde la comunidad es gobernada según sus valores y creencias, prácticas e instituciones (económicas, sociales, culturales y políticas), con derechos y obligaciones colectivas; así como en estructuras democráticas y prácticas de solidaridad y en los principios de reciprocidad, cooperación y confianza. Este enfoque sociocultural busca, en el bienestar colectivo, la afirmación de la identidad cultural, la mejora de las condiciones de vida, y la creación de oportunidades de subsistencia para sus miembros. Este tipo de organizaciones también tiene mecanismos mediante los cuales la titularidad y el manejo de los recursos productivos, así como las riquezas naturales y los ingresos de la organización, son repartidos entre todos los participantes de manera equitativa. (PNUD, 2012) [6].

Según el Instituto Nacional de Estadísticas y Censos (2011), el país registra una población de 14.483 .499 de habitantes, de los cuales el $63 \%$ vive en zonas urbanas y el $37 \%$ en zonas rurales. El 7.4\% se autodefinió montubio, 7.2 afroecuatoriano, $7 \%$ indígena, $71.9 \%$ mestizo y $6.5 \%$ otras autodefiniciones [7].

Sobre esta premisa, el turismo comunitario en general y aquel que se desarrolla en zonas rurales de litoral en particular, merece ser estudiado en función del impacto económico y social que genera en aquellas localidades que se dedican a esta actividad.

Actualmente existen registradas en la FEPTCE 106 comunidades distribuidas en cinco redes que cubren todo el territorio nacional. Entre ellas se encuentra la parroquia rural de Salango, ubicada en Manabí, una de las cinco provincias que conforman el litoral ecuatoriano. (FEPTCE, 2016). Ecuador lleva, así como otros países de la región, alrededor de veinte años en la práctica del turismo comunitario [8].

El objetivo que persigue el estudio es validar factores críticos de éxito turístico en la comunidad de Salango a fin de generar espacios participativos e incluyentes desde las bases mismas de la comunidad, que posibiliten la toma de decisiones y cuyo efecto sea a largo plazo.

\section{Caracterización de Salango.}

En 1996 se crea la parroquia Salango, actualmente con 4.534 habitantes, agrupando en su jurisdicción a las comunas Salango y Las Tunas junto con sus recintos.

La comunidad Salango registra en sus 2.536 hectáreas una población de 3.500 habitantes, de los cuales 480 son comuneros activos, que a diferencia de los no activos, se hacen acreedores a beneficios económicos relacionados con solicitudes de legalización de 
propiedades y de servicios básicos, así como para la participación, como trabajadores en eventos que se organizan en la localidad. Esta comunidad persigue consolidar su proceso de desarrollo considerando al turismo comunitario como uno de los aspectos principales, aprovechando el recurso natural y cultural que posee como una herencia de los pueblos originarios que se asentaron en este sector. Actualmente la pesca es la principal actividad, seguida por la agricultura y el turismo. (Consejo de Gobierno Comunidad Salango, Universidad Estatal del Sur de Manabí, 2018) [9].

Desde 2005 la comunidad Salango asumió la administración del Centro de Investigaciones y Museo Salango aplicando un modelo de manejo comunitario propio. Posteriormente mejoró y complementó la infraestructura turística para brindar el servicio de alojamiento, capacitó a guías nativos, servidores turísticos y reguló la actividad con la aprobación de un reglamento que se encuentra vigente.

Los servicios que ofrece el Centro de Turismo Comunitario Salango - CTCS son los siguientes: Centro de investigaciones y museo, hospedaje comunitario, alimentación, venta de artesanías, tour marino y guías turísticos nativos.

\section{Método.}

A efectos de validar la propuesta de 18 Factores Críticos de Éxito turístico, se empleó el taller como una actividad compartida, de carácter teórico-práctico, caracterizada por ciertos niveles de participación. Rodríguez (2002) [10]. Los habitantes de esta comuna están familiarizados con la dinámica de este método puesto que se han desarrollado actividades de similares características para abordar temas de distinta índole y de interés general, factor que motivó su aplicación.

Se justifica la utilización de este método porque se basa en la experiencia de los participantes, más aún si son miembros de la comunidad; está centrado en los problemas e intereses comunes del grupo. Implica una participación activa de los integrantes y se puede utilizar diversas técnicas, especialmente la discusión racional entre los asistentes.

El taller, como lo cita Careaga (2006) implica un lugar donde se trabaja y se elabora. Es una forma de enseñar y aprender mediante la realización de algo. Es una metodología participativa en la que se enseña y se aprende a través de una tarea conjunta; además, promueve el desarrollo de varios saberes como: cognitivo, operativo y relacional, lo que a su vez se traduce en saber escuchar, planificar con otros, tolerar las opiniones de los demás, aprender a coordinarse con otros, tomar decisiones de manera colectiva, sintetizar, diferenciar entre información relevante y no relevante, factores que hacen del taller un método notable de construcción [11].

Para efectos de desarrollar el taller del 22 de julio de 2018 fue necesario mantener al menos dos reuniones previas con el presidente de la comunidad, Cirilo Macías. Las invitaciones que se cursaron, con quince días de anticipación, estuvieron a cargo del Ministerio de Turismo, siendo así que la coordinadora zonal, Elisa Mora, con quien se mantuvo también una reunión de trabajo anterior, ordenó su diseño. La entrega la formalizó el presidente de la comuna. 


\section{Fases del taller.}

El taller estuvo constituido cronológicamente por las siguientes fases:

1. Presentación del facilitador; 2. Exposición de los objetivos; 3. Presentación de cada participante; 4. Formulación de preguntas iniciales; 5. Entrega del listado de factores críticos; 6. Explicación detallada de cada factor para la correspondiente validación de los participantes; 7. Retroalimentación; 8. Cierre del taller.

Posterior a la presentación del facilitador, se comentó el objetivo del taller que consistía en validar con actores locales la lista de FCE que en determinado momento puede aplicar el CTCS para convertirse en un referente exitoso de turismo comunitario y a continuación cada uno de los participantes expuso sus nombres y apellidos, así como el lugar del cual provenía. Seguidamente los participantes respondieron a la interrogante ¿Qué es el éxito? y luego de escuchar la respuesta de cada uno, se formuló una segunda pregunta ¿Qué factores impiden que el Centro de Turismo Comunitario Salango sea totalmente exitoso? La misma que también fue respondida por cada asistente.

En la fase siguiente el facilitador, utilizando un proyector de imágenes, explicó qué significa factores claves de éxito y su implicación en la actividad turística y expuso cada uno de los 18 factores que resultaron de la adaptación que hicieren los autores del presente estudio sobre aquellos que aparecen en la bibliografía especializada, ante lo cual los asistentes, a quienes se les entregó previamente una hoja con la correspondiente lista de control respondieron si el contenido de cada factor resultaba de significativa importancia o no para el éxito de las actividades de turismo comunitario que desarrolla Salango. Una vez efectuada la tabulación de los datos, se calculó la media y la mediana para interpretar los resultados.

Los factores que se pusieron a consideración de los participantes (ver cuadro 1), resultaron de un proceso de investigación doctoral en curso, desarrollada por uno de los autores en el contexto de ciudades patrimonio mundial (Torres, Martín \& Rodríguez, 2018). Los mismos se definieron en base al método de expertos, tomando en cuenta las singularidades de las ciudades que poseen zonas inscritas en la lista de la UNESCO. Los factores desarrollados están alineados a tres dimensiones del éxito: equilibrio turístico, bienestar de la población local y conservación del patrimonio [12], no obstante, para contextualizar, se procedió a ajustar las dimensiones, orientándolas hacia el turismo comunitario, quedando así: desarrollo turístico equilibrado, conservación del espacio turístico y bienestar de la comunidad.

Identificadas las dimensiones se hizo necesario validar sus factores en el contexto de comunidades de litoral (ver cuadro 1), cuya oferta gira en torno al turismo rural, aspecto que forma parte de un proceso de investigación más amplio en el marco de otra tesis de investigación doctoral, también en curso, llevada a cabo por otro de los autores del presente artículo. 
Cuadro 1. Factores claves de éxito

DIMENSIONES FACTORES CLAVES DE ÉXITO

Efectividad del marketing.- corresponde a las acciones de marketing del destino, si estas son efectivas en términos de posicionamiento y captura de mercados objetivo.

Calidad de la experiencia.- hace referencia a que si el turista que visita la comunidad encuentra una experiencia altamente

DESARROLLO satisfactoria.

TURÍSTICO

EQUILIBRADO

CONSERVACIÓN DEL ESPACIO TURÍSTICO
Gestión turística.- tiene que ver con la eficacia y la pertinencia de las acciones que emprenda la comunidad y si estas posibilitan el éxito, la capacidad de atracción y la sostenibilidad del destino turístico.

Infraestructura turística.- se trata de que si la base para la estructura requerida por los turistas está pensada en favor del desarrollo turístico equilibrado. El $100 \%$ respondió positivamente.

Movilidad sostenible, segura y accesible.- es decir, si el modelo de movilidad no causa impacto negativo sobre las condiciones del medio ambiente y se preocupa, en condiciones de seguridad, por el bienestar y la calidad de vida de todas las personas.

Seguridad turística.- consiste en que las áreas turísticas se perciben libres de riesgo, tanto por el residente local como por el turista.

Cumplimiento de la ley en materia turística.- radica en que si el marco normativo del turismo es acatado y cumplido por el sector comunitario.

Equilibrio funcional.- establece si el desarrollo de la actividad turística evita la invasión agresiva del espacio público y la perturbación del conjunto rural; a la vez se beneficia de un espacio natural y entorno bien mantenidos.

Impactos medioambientales.- es decir, si los impactos medioambientales derivados de la actividad turística se mantienen dentro de límites aceptables.

Estrategias.- relacionada con la interpretación del patrimonio material e inmaterial y si esta se realiza en el marco de una estrategia.

Calidad de interpretación.- tiene que ver con que la interpretación se fundamente en conocimientos, actitud de servicio, competencias comunicacionales e inteligencia emocional, asegurando resultados positivos al turista a nivel de su comprensión, emoción, satisfacción y deseo de visitar otros sitios turísticos. 
BIENESTAR DE LA COMUNIDAD

Empleo.- es decir, si las personas empleadas en el sector turístico reciben una remuneración justa conforme a la ley y reciben cobertura de seguridad social y ocupacional sin discriminación de ninguna naturaleza.

Cohesión e inclusión social.- se ha de identificar si el turismo favorece la tolerancia y solidaridad entre grupos distintos; el acceso libre, sin discriminación, al espacio turístico comunitario de litoral y el sentido de comunidad de los residentes.

Viviendas.- corresponde a que si las personas articuladas a la cadena de valor del turismo en el área, tienen acceso a vivienda de calidad.

Acceso equitativo.- concierne a que si el turismo brinda oportunidades para el emprendimiento y la articulación a la cadena de valor con un enfoque inclusivo, dando acceso al residente local y a las economías informales.

Infraestructura pública básica.- se ha de identificar si la población requiere de servicios básicos para satisfacer sus necesidades vitales, y si estos servicios a su vez favorecen el desarrollo de la actividad turística.

Interacción de actores.- es decir, si se aprecia una participación activa de todos los actores del destino en el establecimiento de estrategias, toma de decisiones y resolución de conflictos.

Impacto económico.- corresponde a que los beneficios de la actividad en la comunidad reflejen que las inversiones en turismo son económicamente beneficiosas y produzcan una rentabilidad social.

Fuente: Torres, Martín y Rodríguez (2018)

\section{Elaboración propia}

Se formuló a los asistentes esta pregunta: ¿Los siguientes factores son de significativa importancia para el éxito de las actividades de turismo comunitario en Salango, sí o no?

Concluida la fase de cumplimentación del cuestionario, los participantes incluyeron en la parte inferior del formulario, tal y como estaba previsto, factores o elementos que desde su particular punto de vista, no se habían previsto en la propuesta. La duración aproximada del taller fue de cuatro horas.

Se contó con la presencia de 12 personas, 9 de ellas hombres y 3 mujeres que representaron a la comunidad Salango y al recinto Rio Chico, perteneciente a la misma comunidad. Al consultar a qué se dedican respondieron: Dirigente de territorio (2), Agricultura (1), Turismo (2), Turismo agroecológico (1), Turismo Comunitario (4), Guía de turismo local (2).

Resultados. 
Ante la pregunta ¿Qué es el éxito?, los participantes indistintamente respondieron: a. Significa alcanzar los objetivos; b. Cumplir con las expectativas esperadas; c. Proyectarse al futuro y alcanzar las metas planteadas; d. Consolidar las capacidades de cada individuo; e. Alcanzar los sueños.

Seguidamente se les consultó ¿Qué factores impiden que el Centro de Turismo Comunitario Salango sea totalmente exitoso? Sus respuestas fueron: a. La falta de fe para invertir; b. El trabajo en equipo aún es escaso; c. El apoyo de las autoridades es insuficiente; d. Las campañas promocionales son débiles; e. El nivel de concienciación de la comunidad es aún bajo; f. Se aprecia ciertos conflictos entre los habitantes; g. No siempre se consulta a las bases para la toma de decisiones.

Siendo que la caracterización de cada factor se presentó en el siguiente orden, a cargo del facilitador, sobre la pregunta ¿Los siguientes factores son de significativa importancia para el éxito de las actividades de turismo comunitario en Salango? ¿Sí o No?, los resultados registrados se los aprecia en el siguiente cuadro.

Cuadro 2. Respuestas

\begin{tabular}{|l|c|c|}
\hline Factor clave de éxito & \% Sí & \% No \\
\hline 1. Efectividad del marketing & 100 & - \\
\hline 2. Calidad de la experiencia & 100 & - \\
\hline 3. Gestión Turística & 100 & - \\
\hline 4. Infraestructura turística & 100 & - \\
\hline 5. Movilidad sostenible, segura y accesible & 66,7 & 33,3 \\
\hline 6. Seguridad Turística & 100 & - \\
\hline 7. Cumplimiento de la ley en materia turística & 91,6 & 8,4 \\
\hline 8. Equilibrio funcional & 91,6 & 8,4 \\
\hline 9. Impactos medioambientales & 100 & - \\
\hline 10. Estrategias & 100 & - \\
\hline 11. Calidad de interpretación & 100 & - \\
\hline 12. Empleo & 100 & - \\
\hline 13. Cohesión e inclusión social & 100 & - \\
\hline 14. Viviendas & 100 & - \\
\hline 15. Acceso equitativo & 100 & - \\
\hline 16. Infraestructura pública básica & 100 & - \\
\hline 17. Interacción de actores & 100 & - \\
\hline 18. Impacto económico & 100 & - \\
\hline
\end{tabular}

Tabulados que fueran estos resultados, la media corresponde al $97,21 \%$ y la mediana al $100 \%$.

En la parte final se solicitó que, en caso de estimarlo conveniente, los participantes incluyan factores que no hayan sido considerados, ante lo cual dos de ellos expresaron: a. Gestión con gobiernos amigos; b. Perseverancia en los proyectos turísticos. 


\section{Discusión.}

El taller brindó la oportunidad de expresar libremente criterios de los asistentes como sucede con las variables que posibilitarían optimizar la gestión que lleva a cabo el Centro de Turismo Comunitario Salango a través de una herramienta de gestión estratégica (FCE) que viabilice, sobre base del éxito de esta localidad turística, mejorar las condiciones del buen vivir de las personas que allí habitan.

Las reacciones de los comuneros frente a las dos primeras preguntas abiertas dejan ver que la capacidad de discernir está latente ante los temas abordados, es decir, el éxito y sus limitaciones, así como en el análisis de cada factor clave de éxito puesto a consideración del público cuyo resultado corresponde en promedio al 97,21\% de aceptación; se muestra la necesidad de sortear variables internas y externas que permitan vigorizar las acciones que lleva a cabo el CTCS con el involucramiento de toda la comunidad en el logro de los objetivos y las metas de la organización, hasta la participación de gobiernos extranjeros para estos mismos propósitos, puesto que son varias las acciones que se han desarrollado con la colaboración de organizaciones no gubernamentales, nacionales y extranjeras en la zona sur de Manabí, elemento que motivó a los participantes a recomendar su inclusión como un factor crítico de éxito más.

\section{Conclusiones.}

Los resultados que se derivaron del presente estudio, permitieron emitir las siguientes conclusiones:

$>$ El éxito, para los miembros del CTCS se traduce en el logro de objetivos y metas planteados por la organización, así como, alcanzar las expectativas sobre base del trabajo individual y colectivo de sus habitantes.

$>$ Las organizaciones turísticas que gestionan el turismo comunitario se ven obligadas a plantear alternativas innovadoras que les permitan sacar ventaja del entorno natural y cultural en el cual se desarrollan y a su vez procurar una alternativa disímil a la que promete el turismo tradicional.

$>$ El promedio de aprobación del 97,21\% de los factores claves de éxito por parte de representantes del Centro de Turismo Comunitario Salango demuestra que su aplicación a corto plazo generaría un impacto más significativo para las condiciones de la prestación de servicios.

$>$ La participación activa de la comunidad en la toma de decisiones se torna concluyente si el propósito es ofrecer productos turísticos con enfoque comunitario, más aún si gran parte del control y sus beneficios, corresponde a la mayoría de sus habitantes.

$>$ Entre los principales resultados de la investigación y que no fueron considerados en la propuesta de factores claves de éxitos, se determina que los conflictos internos que se generen en la comunidad, podrían convertirse en limitantes al momento de poner en marcha nuevas iniciativas de servicios que brinde el CTCS. 
La participación de gobiernos amigos es una posibilidad real al momento de proyectar objetivos y estrategias de diversificación de la oferta turística que brinda el CTCS.

$>$ El rol de algunos de los actores, específicamente el de las autoridades, no es lo suficientemente consistente al momento de emprender acciones concretas relacionadas con el fortalecimiento de la gestión que lleva a cabo el CTSC.

\section{Referencias Bibliográficas.}

TORRES MATOVELLE, Pablo. Índice de éxito turístico en ciudades destino. Revista Estudios y Perspectivas en Turismo, [en línea] 27: 431- 445, 2018. [Consulta: 24 de agosto de 2018]. Disponible en: www.estudiosenturismo.com.ar/ ISSN 18511732.

KAPLAN, R. \& NORTON, D. The Balanced Scorecard: Measures that Drive Performance. Harvard Business Review, 1992. 70(1): 71-79. https://hbr.org/magazine ISSN 1851-1732.

ALFONSO FERRERAS, Víctor. Tesis: Un modelo integral para evaluar la competitividad de destinos turísticos basado en la identificación e integración de factores críticos de éxito (en opción al grado científico de Doctor en Turismo y Desarrollo Sostenible). Universidad de Las Palmas de Gran Canaria, España. 2009.

DE FREITAS, Vidalina; YABER, Guillermo. GECONTEC: Revista Internacional de Gestión del Conocimiento y la Tecnología. ISSN 2255-5684 Vol.3 (1). 2015.

CONSEJO NACIONAL DE PLANIFICACIÓN. Plan Nacional del Buen Vivir 2017 2021. Quito, Ecuador.

UNITED NATIONS DEVELOPMENT PROGRAMME. Multinational Federation of Community Tourism in Ecuador (FEPTCE) 2012. Equator Initiative Case Study Series. New York, NY.

INSTITUTO NACIONAL DE ESTADÍSTICAS Y CENSOS. Informe del Censo de población y vivienda. 2011. Quito - Ecuador.

FEDERACIÓN PLURINACIONAL DE TURISMO COMUNITARIO DEL ECUADOR FEPTCE. Informativo anual. 2016. Quito - Ecuador.

RODRÍGUEZ LUNA, María Elvira. Tesis: Cualificación de docentes y desarrollo de estrategias argumentativas en los niños de preescolar en el marco de la pedagogía de proyectos. Una propuesta fundamentada en el análisis de la interacción en el aula. (En opción al grado científico de Doctor en Educación. Énfasis de Lenguaje, Universidad Distrital Francisco José de Caldas. Colombia. 2002. 
CAREAGA, Adriana., SICA, Rosario., CIRILLO, Ángela., DA LUZ, Silvia. Aportes para diseñar e implementar un taller. 8vo. Seminario -Taller en Desarrollo Profesional Médico Continuo (DPMC) 2das Jornadas de Experiencias educativas en DPMC. Octubre 5,6 y 7, 2006. México.

TORRES, Pablo., MARTÍN, Ramón. \& RODRÍGUEZ, Vilma. Índice para la medición del éxito turístico en una ciudad patrimonio de la humanidad. Ponencia presentada en el XVI Encuentro Internacional sobre Gestión de Ciudades Patrimoniales. 2018. La Habana, Cuba.

CONSEJO DE GOBIERNO COMUNIDAD SALANGO., Universidad Estatal del Sur de Manabí. Plan de Desarrollo Integral de la Comunidad Salango. 2018. Salango, Manabí. 


\section{Para citar el artículo indexado.}

Álvaro G., Torres P. \& Fernández R. (2019) Factores críticos de éxito turístico para comunidades asentadas en zonas de litoral. Caso de estudio: Comunidad de Salango, provincia de Manabí, Ecuador. Revista electrónica Explorador Digital 3(3), 26-37. Recuperado desde:

http://cienciadigital.org/revistacienciadigital2/index.php/exploradordigital/article/view/441/9 $\underline{93}$

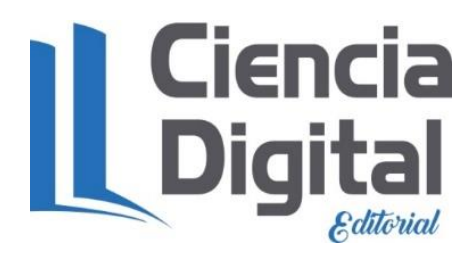

El artículo que se publica es de exclusiva responsabilidad de los autores y no necesariamente reflejan el pensamiento de la Revista Explorador Digital.

El articulo queda en propiedad de la revista y, por tanto, su publicación parcial y/o total en otro medio tiene que ser autorizado por el director o editor de la Revista Explorador Digital.
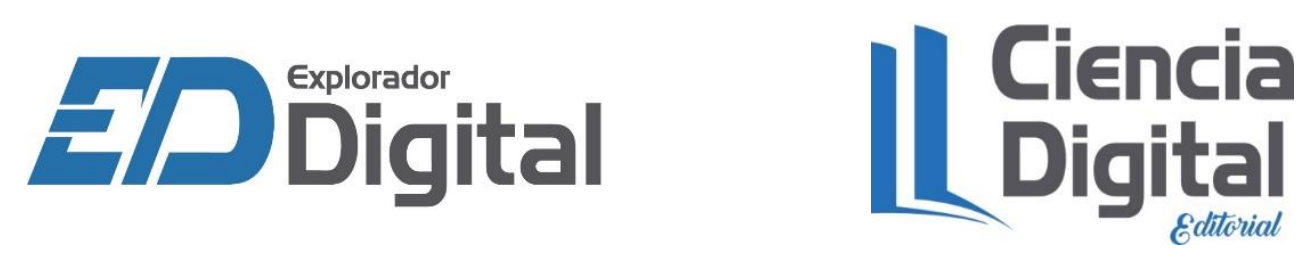